\title{
Effect of dummy vias on interconnect temperature variation
}

\author{
WANG Zeng*, DONG Gang, YANG YinTang \& LI JianWei \\ Key Laboratory of the Ministry of Education for Wide Band-Gap Semiconductor Materials and Devices, Microelectronics Institute, Xidian Uni- \\ versity, Xi'an 710071, China
}

Received December 1, 2010; accepted January 21, 2011

\begin{abstract}
The number of the dummy via can significantly affect the interconnect average temperature. This paper explores the modeling of the interconnect average temperature in the presence of multiple dummy vias. The proposed model incorporates the multi-via effect into the effective thermal conductivity of the interlayer dielectric (ILD) to obtain accurate results. Using different ILDs, the multi-via effect is analyzed and discussed. Also, the extended applications of the multi-via effect are presented in this paper to obtain the minimum interconnect average temperature increase with a given via separation or number. This study suggests that the multi-via effect should be accounted for in integrated circuits design to optimize the performance and design accuracy of integrated circuits.
\end{abstract}

interconnect modeling, interconnect average temperature increase, multi-via effect, dummy via, interlayer dielectric

Citation: Wang Z, Dong G, Yang Y T, et al. Effect of dummy vias on interconnect temperature variation. Chinese Sci Bull, 2011, 56: 2286-2290, doi: $10.1007 / \mathrm{s} 11434-011-4414-2$

As feature sizes have decreased, a continuous increase in integration density has resulted in higher overall chip temperature, which makes power dissipation and thermal issues a major impediment in the design of ultra large scale integrated circuits (ULSI) [1-3]. Research has shown that on-chip global interconnect self-heating contributes about $34 \%$ of the total chip power dissipation in an Intel microprocessor with 130-nm features [4]. Moreover, because of the reduction of the distance between the substrate and the top-most metal layers [5], the substrate temperature variations have an increased effect on interconnect temperature profile. In high-performance integrated circuits (ICs), the peak chip temperature can reach $210^{\circ} \mathrm{C}$ and the thermal gradients can rise above $50^{\circ} \mathrm{C}$ when in the $\mathrm{GHz}$ frequency regime [6,7]. The temperature distribution can strongly affect the interconnect resistance, and, consequently, the signal integrity of circuits can be degraded.

Furthermore, low-k materials are generally used to reduce the effect on interconnect time delay, crosstalk and dynamic power consumption $[8,9]$. However, because of the poor thermal conductivity of such materials, most of the

*Corresponding author (email: fire5water@ hotmail.com)
Joule heating produced by the interconnects on every level cannot be transmitted to the heat sink. Therefore it accumulates which leads to a larger interconnect temperature rise. However, vias can effectively reduce interconnect temperature increase. Hence the interconnect temperature will not be not as high would be estimated or the previous circumstance [10-13]. Because of the reasonable thermal conductivity of vias, dummy vias can be introduced to reduce the interconnect temperature in IC design. A dummy via is a kind of via which that used to conduct heat but is not electrically connected [12-15]. The interconnect temperature profile can be strongly affected by the presence of dummy vias. Over-estimation of the interconnect temperature can lead to design failure or unnecessarily conservative design. Therefore, an accurate prediction of interconnect temperature is a necessary precondition for interconnect characterization.

\section{Interconnect temperature model with multiple dummy vias}

For our model, we assumed that an interconnect exchanges heat with the environment only through the substrate [5]. In 
addition, as shown in Figure 1, for a global interconnect, the temperature in the length direction varies more quickly than in the directions of thickness and width. Therefore, we take only the temperature variation in the length direction into account when solving the equation.

Also, we assume the temperature at interconnect terminals connected by vias is the same as the constant underlying layer temperature, $T_{\text {ref }}$. Therefore, the temperature distribution along an interconnect with thickness $t_{m}$, width $w_{m}$ and length $L$ can be formulated by the following heat diffusion equation [12]:

$$
\begin{aligned}
& k_{m} \frac{\mathrm{d}^{2} T(x)}{\mathrm{d} x^{2}}+j_{\mathrm{rms}}^{2} \rho_{0}\left[1+\beta\left(T(x)-T_{r e f}\right)\right] \\
& -j_{\mathrm{rms}} \varepsilon \frac{\mathrm{d} T(x)}{\mathrm{d} x}-\frac{k_{i n s} w^{*}}{t_{i n s} t_{m} w_{m}}\left(T(x)-T_{r e f}\right)=0,
\end{aligned}
$$

where $T(x)$ represents the temperature profile of the interconnect. $k_{m}$ and $k_{i n s}$ are the thermal conductivity of the interconnect and the underlying interlayer dielectric (ILD), respectively. $j_{\mathrm{rms}}$ is the uniform root-mean-square current density flowing in the interconnect. $\rho_{o}$ is the resistivity of the interconnect at the reference temperature, $T_{\text {ref }} . \beta$ is the temperature coefficient of resistance $\left({ }^{\circ} \mathrm{C}^{-1}\right) . \varepsilon$ is the Thomson coefficient. The third term represents the heat loss resulting from the Thomson effect, which is very small and is neglected here. The forth term is the energy loss due to the heat transmission between the adjacent interconnects through the insulator. $t_{i n s}$ is the thickness of the ILDs. $w^{*}$ is the effective interconnect width for thermal diffusion. By solving eq. (1), we can obtain:

$$
T(x)=T_{r e f}+\frac{j_{\text {rms }}^{2} \rho_{0}}{k_{m} \alpha^{2}}\left[1-\frac{\cosh (\alpha x)}{\cosh (\alpha L / 2)}\right],-L / 2 \leqslant x \leqslant L / 2,
$$

where

$$
\alpha=\sqrt{\frac{k_{\text {ins }} w^{*}}{t_{\text {ins }} t_{m} w_{m} k_{m}}-\frac{j_{\mathrm{rms}}^{2} \rho_{0} \beta}{k_{m}}},
$$

because the fluctuation of the resistivity due to the tem-

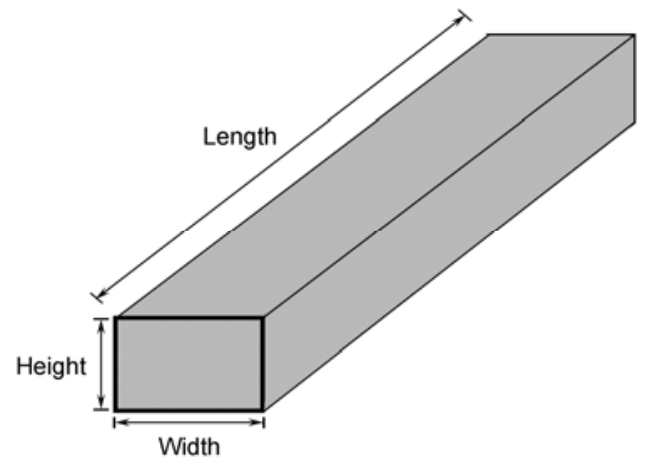

Figure 1 A global interconnect schematic diagram. perature fluctuation is small enough to be ignored [12], $\alpha$ can be simplified to $\alpha=\sqrt{\frac{k_{\text {ins }} w^{*}}{t_{\text {ins }} t_{m} w_{m} k_{m}}}$.

It is assumed that the number of the vias in the interconnect of interest is $n$. Because of the via effect, the interconnect average temperature increase $T_{\text {avg }}$ can be expressed as the total temperature increase divided by the interconnect length, $L$ :

$$
T_{\text {avg }}=\frac{1}{L} \sum_{i=2}^{n} \int_{l_{i}}\left[T_{n}(x)-T_{\text {ref }}\right] \mathrm{d} x,
$$

where $T_{n}(x)$ is the temperature profile between via $n-1$ and via $n$. $L$ is the total interconnect length, $l_{i}$ is the distance between via $i-1$ and via $i$. Let the number of the vias be even (eight vias for example) and the vias in the cooling area are equidistantly arranged. The detailed temperature increment with multiple dummy vias is shown in Figure 2.

Let $T_{l}$ be the average temperature increase of the main interconnect, and let $T_{v}$ be the average temperature increase of the interconnect of length $l$ between adjacent vias in the cooling area. The average temperature increase $T_{l}$ and $T_{v}$ can be expressed as

$$
\begin{gathered}
T_{l}=\frac{j_{\mathrm{rms}}^{2} \rho_{0}}{k_{m} \alpha^{2}} \int_{-\frac{L}{2}+\frac{L-2}{2} l}^{\frac{L}{2}-\frac{n-2}{2} l}\left[1-\frac{\cosh (\alpha x)}{\left.\cosh \left(\frac{1}{2} \alpha(L-n l+2 l)\right)\right]} \mathrm{d} x\right. \\
=\frac{j_{\mathrm{rms}}^{2} \rho_{0}[L-(n-2) l]}{k_{m} \alpha^{2}}\left[1-\frac{\tanh \left(\frac{1}{2} \alpha(L-n l+2 l)\right)}{\frac{1}{2} \alpha(L-n l+2 l)}\right], \\
T_{v}=\frac{j_{\mathrm{rms}}^{2} \rho_{0}}{k_{m} \alpha^{2}} \int_{-\frac{l}{2}}^{\frac{l}{2}}\left[1-\frac{\cosh (\alpha x)}{\left.\cosh \left(\frac{1}{2} \alpha l\right)\right] \mathrm{d} x}\right] \\
=\frac{j_{\mathrm{rms}}^{2} \rho_{0} l}{k_{m} \alpha^{2}}\left[1-\frac{\left.\tanh \left(\frac{1}{2} \alpha l\right)\right]}{\frac{1}{2} \alpha l}\right] .
\end{gathered}
$$

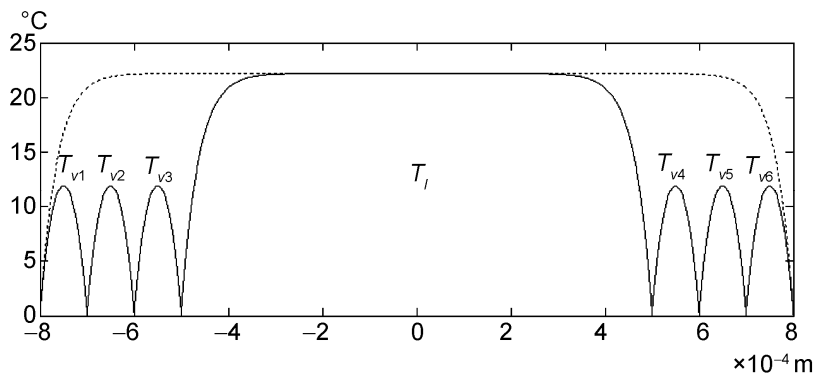

Figure 2 Temperature profile for an interconnect with multiple vias. The solid line represents the temperature profile of an interconnect that has multiple dummy vias, and the dotted line represents the thermal distribution of the same interconnect with only two vias. 
temperature increase, $T_{\text {avg }}$, can be expressed as

$$
\begin{aligned}
& T_{\text {avg }}=\frac{1}{L}\left[T_{l}+(n-2) T_{v}\right] \\
& =\frac{j_{r m s}^{2} \rho_{0}}{k_{m} \alpha^{2} L}\left\{\begin{array}{c}
L-\frac{\tanh \left[\frac{1}{2} \alpha(L-(n-2) l)\right]}{\frac{1}{2} \alpha} \\
-(n-2) \frac{\tanh \left(\frac{1}{2} \alpha l\right)}{\frac{1}{2} \alpha}
\end{array}\right\} .
\end{aligned}
$$

\section{Effect of multiple dummy vias on thermal conductivity of the ILDs}

Dummy vias have different effects on the average temperature increase for different ILDs [12]. To obtain the correct interconnect temperature increase, the effect of multiple dummy vias can be incorporated into the effective thermal conductivity during the analysis of interconnect characteristics. The average interconnect temperature increase can be expressed as

$$
T_{\text {avg }}^{*}=j_{\text {thermal }} R_{\text {thermal }}=\frac{j_{\mathrm{rms}}^{2} \rho_{0} t_{m} t_{\text {ins }} w}{k_{\text {ins }}^{*} w^{*}},
$$

where $R_{\text {thermal }}$ represents the thermal resistance of the interconnect. $j_{\text {thermal }}$ is the heat transfer rate. $k_{\text {ins }}^{*}$ is the effective thermal conductivity of ILD. If we set eqs. (7) and (8) to be equal:

$$
T_{\text {avg }}=T_{\text {avg }}^{*},
$$

and solving eq. (9), we obtain:

$$
k_{i n s}^{*}=k_{i n s}\left\{\begin{array}{r}
\tanh \left[\frac{1}{2} \alpha(L-n l+2 l)\right] \\
1-\frac{-(n-2) \tanh \left(\frac{1}{2} \alpha l\right)}{\frac{1}{2} \alpha L}
\end{array}\right\} .
$$

We define $\xi$ as the dummy via correction coefficient, and it is expressed as

$$
\xi=\left\{\begin{array}{r}
\tanh \left[\frac{1}{2} \alpha(L-n l+2 l)\right] \\
1-\frac{-(n-2) \tanh \left(\frac{1}{2} \alpha l\right)}{\frac{1}{2} \alpha L}
\end{array}\right\}^{-1} .
$$

Therefore,

$$
k_{\text {ins }}^{*}=k_{\text {ins }} \xi .
$$

Using eq. (11) we find that the value of $\xi$ is larger than 1 , and it increases as $n$ becomes larger. Accordingly, the low-k dielectrics become more thermally conductive, because of the presence of the dummy vias. Table 1 shows the effect of the dummy via number on the effective thermal conductivity for different ILDs.

Table 1 shows the multi-via effect on the conductivity for three kinds of dielectrics, $\mathrm{SiO}_{2}$, Orion and air. From the table it can be seen that the lower the nominal thermal conductivity, the more the via number effects the effective thermal conductivity of a low-k dielectric. When $n=10$, the effective thermal conductivity of the air becomes 0.064 $\mathrm{W} / \mathrm{m} \mathrm{K}$, which is almost twice its nominal thermal conductivity. Also, the effective thermal conductivity is doubled when $n=30$ for Orion and when $n=78$ for $\mathrm{SiO}_{2}$.

The relationship between the interconnect average temperature increase $\left({ }^{\circ} \mathrm{C}\right)$ and the via number for different ILDs is shown in Table 2, where

$$
\eta=\frac{T_{\text {avg }}(n=2)-T_{\text {avg }}(n>2)}{T_{\text {avg }}(n=2)} \times 100 \% .
$$

From Table 2, it can be seen that the interconnect average temperature increase for ILDs with low thermal conductivities are larger than those for ILDs with high thermal conductivities. However, the via effect is more sensitive to $n$ for low-k dielectrics. For example, with air as the ILD, the interconnect average temperature increase is $105.13^{\circ} \mathrm{C}$ when $n=2$, and it is $43.60^{\circ} \mathrm{C}$ when $n=12$, which is less

\begin{tabular}{|c|c|c|c|c|c|c|c|c|c|c|c|c|}
\hline & $n=2$ & Change rate $(\%)$ & $n=4$ & Change rate $(\%)$ & $n=6$ & Change rate $(\%)$ & $n=8$ & Change rate $(\%)$ & $n=10$ & Change rate $(\%)$ & $n=12$ & Change rate $(\%)$ \\
\hline$k_{\mathrm{SiO}_{2}}$ & 1.055 & 1.44 & 1.069 & 2.79 & 1.083 & 4.13 & 1.098 & 5.58 & 1.113 & 7.02 & 1.129 & 8.56 \\
\hline$k_{\text {Orion }}$ & 0.166 & 3.75 & 0.173 & 8.13 & 0.179 & 11.86 & 0.186 & 16.25 & 0.194 & 21.25 & 0.202 & 26.25 \\
\hline
\end{tabular}
than $50 \%$ of the original temperature increment. This is the reason why the interconnect average temperature increase is not as high as commonly estimated when low- $k$ materials are used in advanced interconnect structures. As a result, increasing the number of dummy vias is a simple and effective way to decrease the interconnect average temperature

Table 1 Influence of the dummy via number on the effective thermal conductivity for different ILDs ${ }^{\text {a) }}$

a) The unit of thermal conductivity is $\mathrm{W} \mathrm{m}^{-1} \mathrm{~K}^{-1}$; the nominal thermal conductivities of $\mathrm{SiO}_{2}$, Orion and air are $1.04,0.16$ and $0.03 \mathrm{~W} \mathrm{~m}^{-1} \mathrm{~K}^{-1}$, respectively [13]. 
increase.

\section{Extension of the application of the multi-via effect}

When $n$ is constant and $l=L /(n-1)$, the interconnect average temperature increase is minimized. The minimum interconnect average temperature increase is given as follows:

$$
T_{\text {avg_min }}=\frac{j_{\text {rms }}^{2} \rho_{0}}{k_{m} \alpha^{2}}\left[1-(4 n-8) \frac{\tanh \left(\frac{\alpha L}{2 n-2}\right)}{\alpha L}\right] .
$$

Interconnect average temperature increase is dependent on the separation of the dummy vias. Figure 3 shows the relationship between the interconnect average temperature increase and via separations for 65-nm technology [16].

Let $n$ be 11, and let the interconnect length be $2000 \mu \mathrm{m}$. We used seven different via separations, namely, 50, 80, $100,150,200,210$ and $220 \mu \mathrm{m}$, to analyze the effect of via separation on interconnect average temperature increase. From Figure 3, it can be seen that the minimum interconnect average temperature increase happens at $l=200 \mu \mathrm{m}$. It can also be seen that the change of interconnect average temperature increase varies smoothly over the range $l=$ 80-210 $\mu \mathrm{m}$ for an $\mathrm{SiO}_{2}$ ILD. However, the interconnect average temperature increase changes significantly for different via separations as the thermal conductivity is reduced, which is shown in Figure 3(b) and (c). As a result, for a constant number of the dummy vias, we can adjust the via separation to reduce the interconnect temperature increase and decrease the power dissipation.

For a given $l$, using eq. (7), the interconnect average temperature increase is minimized when

$$
n=\left[\begin{array}{l}
\frac{\alpha L+2 \alpha l+}{\tanh ^{-1}\left[\frac{\sqrt{\alpha l\left(\mathrm{e}^{\alpha l}+1\right)\left(-2 \mathrm{e}^{\alpha l}+2+\alpha l \mathrm{e}^{\alpha l}+\alpha l\right)}}{\alpha l\left(\mathrm{e}^{\alpha l}+1\right)}\right]} \\
\alpha l
\end{array}\right] .
$$

For a constant interconnect length $(l=200 \mu \mathrm{m})$, the number of the dummy vias vs. the interconnect average temperature increase is shown in Figure 4.

In Figure 4, it can be seen that the average interconnect temperature increase has an approximately linear relationship with via number. The increased the thermal conductivity of the ILD is, the more linear the relation is. Therefore, the via number can be estimated by the calculated temperature increase for a constant via separation.

\section{Conclusion}

A novel analytical model for analyzing the interconnect average temperature increase in the presence of multiple vias is developed in this paper. The proposed model incorporates the multi-via effect into the thermal conductivity of the ILD to obtain a more accurate calculation of the interconnect average temperature increase. For a number of different ILDs, this paper discusses the interconnect average temperature increase in terms of the multi-via effect. We concluded that the multi-via effect has a significant effect on the effective thermal conductivity of the ILD. Moreover, two extended applications of the proposed model are presented. Using these applications, we can minimize the interconnect average temperature increase to decrease the power dissipation of the interconnect. The proposed model can help IC designers estimate the interconnect average temperature increase, modify the interconnect arrangement and obtain the expected interconnect average temperature increase. Likewise, the proposed model with the multi-via

Table 2 Multi-via effect on the interconnect temperature increase for different ILDs

\begin{tabular}{rrrrrrrrrrrr}
\hline & $n=2$ & $n=4$ & $\eta(\%)$ & $n=6$ & $\eta(\%)$ & $n=8$ & $\eta(\%)$ & $n=10$ & $\eta(\%)$ & $n=12$ & $\eta(\%)$ \\
\hline$\Delta T_{\mathrm{SiO}_{2}}$ & 3.37 & 3.32 & 1.48 & 3.28 & 2.67 & 3.24 & 3.86 & 3.19 & 5.34 & 3.15 & 6.53 \\
$\Delta T_{\text {Orion }}$ & 21.38 & 20.62 & 3.55 & 19.85 & 7.16 & 19.09 & 10.71 & 18.32 & 14.31 & 17.56 & 17.87 \\
$\Delta T_{\text {Air }}$ & 105.13 & 92.82 & 11.71 & 80.51 & 23.42 & 68.21 & 35.12 & 55.91 & 46.82 & 43.60 & 58.53 \\
\hline
\end{tabular}
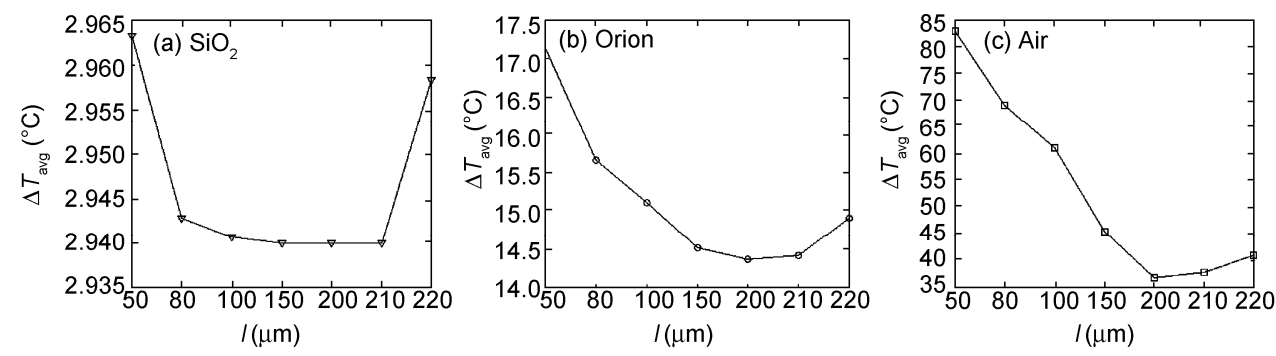

Figure 3 Interconnect average temperature increase for different via separations at constant $n$. 

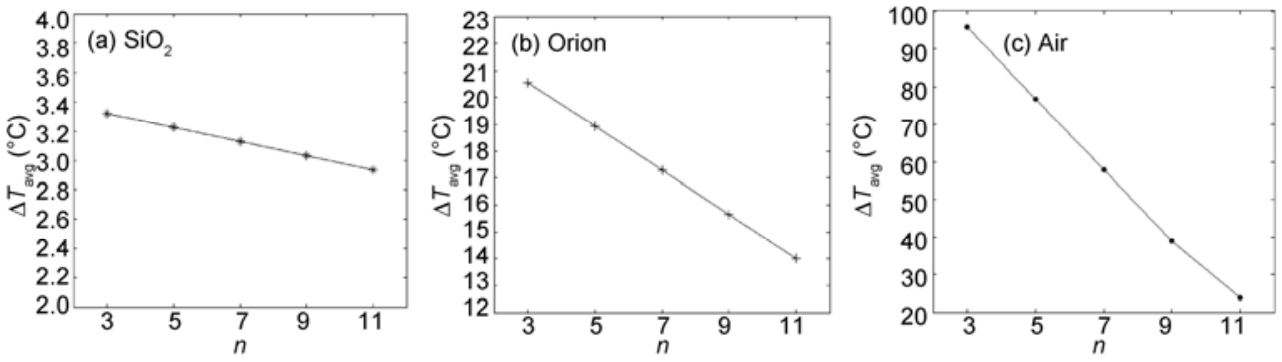

Figure 4 Relationship between the interconnect average temperature increase and via number for a constant $l$.

effect can also be used to research and improve models of interconnect delay, crosstalk and power dissipation.

This work was supported by the National Natural Science Foundation of China (60606006), the National Science Fund for Distinguished Young Scholars of China (60725415) and the Basic Science Research Fund of Xidian University.

1 Subrina S, Kotchetkov D, Balandin A A. Heat removal in silicon-on-insulator integrated circuits with graphene lateral heat spreaders. IEEE Electron Device Lett, 2009, 30: 1281-1283

2 Wang Z, Dong G, Yang Y T, et al. Crosstalk noise voltage of coupling RC interconnect with temperature distribution. Chinese J Electron, 2010, 19: 43-47

3 Wang Z, Dong G, Yang Y T, et al. Study on clock skew of unsymmetrical RLC interconnect tree with temperature distribution (in Chinese). Acta Phys Sin, 2010, 59: 5646-5651

4 Sundaresan K, Mahapatra N R. An analysis of timing violations due to spatially distributed thermal effects in global wires. In: Levitan P S, Fix L, eds. Proceedings of ACM/IEEE DAC, 2007 June 4-8, San Diego. New York: Association for Computing Machinery Press, 2007. 515-520

5 Im S, Banerjee K. Full chip thermal analysis of planar (2-D) and vertically integrated (3-D) high performance ICs. In: Marek J, Illing M, eds. IEEE Int Electron Devices Meeting, 2000 Dec 10-13, San Francisco. Hong Kong: The University of Hong Kong Press, 2001. 727-730

6 Ni M, Memik S O. Self-heating-aware optimal wire sizing under Elmore delay model. In: Lauwereins R, Madsen J, eds. Design, Automation \& Test in Europe Conference \& Exhibition, 2007 April 16-20, Nice. France: Springer-Verlag, 2007. 1-6

7 Datta B, Burleson W P. Low power on-chip thermal sensors based on wires. In: Mooney V, Zhao L, Hasler P, eds. IFIP Int Conf on Very Large Scale Integration, 2007 Oct 15-17, Atlanta. New York:
Springer-Verlag, 2008. 258-263

8 Che F X, Zhang X, Zhu W H, et al. Reliability evaluation for copper/low-k structures based on experimental and numerical methods. IEEE Trans Dev Mater Relia, 2008, 8: 455-463

9 Talanov V V, Schwartz A R. Near-field scanning microwave microscope for interline capacitance characterization of nanelectronics interconnect. IEEE Trans Micro Theory Tech, 2009, 57: 1224-1229

10 Zhu Z M, Li R, Hao B T, et al. A novel analytical thermal model for multilevel nano-scale interconnects considering the via effect. Chin Phys B, 2009, 18: 4995-5000

11 Wang N L, Zhou R D. A novel analytical thermal model for temperature estimation of multilevel ULSI interconnects. In: Huang R, Yu M, Juin J L, eds. Int Conf on Solid-State and Integrated Circuits Technology, 2004 Oct 18-21, Beijing, China. Beijing: Institute of Electrical and Electronics Engineers Press, 2005. 1088-1091

12 Chiang T Y, Banerjee K, Saraswat K C. Compact modeling and spice-based simulation for electrothermal analysis of multilevel ULSI interconnects. In: Hassoun S, Gielen G, Pedram M, eds. Proc Int Conf on Computer-Aided Design, 2001 Nov 4-8, San Jose. New York: Institute of Electrical and Electronics Engineers Press, 2002. 165-172

13 Zhu Z M, Hao B T, Qian L B, et al. A compact interconnect temperature distribution model considering the via effect and the heat fringing effect (in Chinese). Acta Phys Sin, 2009, 58: 7130-7135

14 Ajami A H, Banerjee K, Pedram M. Modeling and analysis of nonuniform substrate temperature effects on global ULSI Interconnects. IEEE Trans Computer-Aided Design of Integrated Circuits and Systems, 2005, 24: 849-861

15 Chiang T Y, Shieh B, Saraswat K C. Impact of Joule Heating on Scaling of Deep Sub-Micron Cu/ow-k Interconnects. In: Taur Y, Maeguchi K, eds. IEEE Int Symp VLSI Technology, 2002 June 11-13, Honolulu. Gaithersburg: Widerkehr and Associates Press, 2002. 38-39

16 ESIA, JEITA, KSIA, et al. International technology roadmap for semiconductors. Technical Report, ITRS for Semiconductors 2007 edition: Interconnect, the ITRS Organization. 2007

Open Access This article is distributed under the terms of the Creative Commons Attribution License which permits any use, distribution, and reproduction in any medium, provided the original author(s) and source are credited. 